\title{
DUALITY AND ASYMPTOTIC SOLVABILITY OVER CONES ${ }^{1}$
}

BY A. BEN-ISRAEL, A. CHARNES AND K. O. KORTANEK

Communicated by I. N. Herstein, October 14, 1968

Introduction. A duality theory for linear programming over closed convex cones is developed by using the solvability theorem of [1]. A complete classification of all duality states is given, which includes Duffin's duality theorem (1 of [3]), together with asymptotic refinements. For convenience, our results are stated in $R^{n}$, but extensions to more general spaces considered in [1] are possible. Complete proofs of all results will be given elsewhere. The main ingredients of the duality theory over closed convex cones are essentially due to Duffin as set forth in his fundamental paper [3]; see also Kretschmer [5].

0. Notations and preliminaries. $C$-a closed convex cone in $R^{n}$,

$$
C^{*}=\left\{y \in R^{n}: x \in C \Rightarrow(y, x) \geqq 0\right\} .
$$

For the system

$$
A x=b \quad x \in C
$$

with given $A \in R^{m \times n}, b \in R^{m}$ let

$$
\begin{gathered}
F(1)=\{x \in C: A x=b\}, \\
A F(1)=\left\{\left\{x_{k}: k=1,2, \cdots\right\}: x_{k} \in C, \lim _{k} A x_{k}=b\right\} .
\end{gathered}
$$

$A F(1)$ is a set of sequences. If $F(1) \neq \varnothing$ then it can be imbedded naturally in $A F(1)$.

DEFINITION. (1) is

CONS (consistent) if $F(1) \neq \varnothing$,

INC (inconsistent) if $F(1)=\varnothing$,

AC (asymptot. consist.) if $A F(1) \neq \varnothing$,

SINC (strongly inconsist.) if $A F(1)=\varnothing$.

We use the following

1 This research was partly supported by the National Science Foundation, Project GP 7550, and by the Office of Naval Research, Contract 1228(10) No 14-67-A0356-0006, and by the U. S. Army Research Office-Durham, Contract No. DA-31124-ARO-D-322, at Northwestern University and National Science Foundation Grant NSF-GP 2729 at Cornell University. Reproduction of this paper in whole or in part is permitted for any purpose of the United States Government. 
Solvability THEOREM ([1, p. 543]). The following are equivalent:

(i) $A x=b, x \in C$ is $A C$,

(ii) $A^{t} y \in C^{*} \Rightarrow(b, y) \geqq 0$.

Consider now the pair of problems

(I.C) $\sup (c, x)$ subject to $A x=b, x \in C$,

(II.C*) $\inf (b, y)$ subject to $A^{t} y-c \in C^{*}$

with given $A \in R^{m \times n}, b \in R^{m}, c \in R^{n}$ and the associated systems

$$
\begin{gathered}
A x=b, \quad x \in C, \\
\left(A^{t},-I\right)\left(\begin{array}{l}
y \\
z
\end{array}\right)=c,
\end{gathered}
$$

(2)

$$
\left(\begin{array}{l}
y \\
z
\end{array}\right) \in R^{m} \times C^{*}
$$

Definition. $^{2}$ (i) (I.C) [(II.C*)] is CONS, INC, AC or SINC if so is $(1)[(2)]$.

(ii) Let (1) $[(2)]$ be AC. Then (I.C) $\left[\left(\mathrm{II} . \mathrm{C}^{*}\right)\right]$ is PAC (properly $\mathrm{AC})$ if $\exists\left\{x_{k}\right\} \in A F(1) \ni \lim _{k}$ sup $\left(c, x_{k}\right)>-\infty\left[\left\{\left(y_{k}, z_{k}\right)\right\} \in A F(2)\right.$ $\left.\ni \lim _{k} \inf \left(b, y_{k}\right)<\infty\right]$; otherwise it is IAC (improperly AC).

(iii) Let (I.C) $[($ II.C*)] be CONS. Then it is BD (bounded) if $\sup \{(c, x): x \in F(1)\}<\infty[\inf \{(b, y):(y, z) \in F(2)\}>-\infty]$. Otherwise it is UBD (unbounded).

(iv) Let (I.C) [(II.C*)] be PAC. Then it is ABD (asymptotically BD) if $\sup \left\{\lim _{k} \sup \left(c, x_{k}\right):\left\{x_{k}\right\} \in A F(1)\right\}<\infty$

$$
\text { [inf } \left.\left\{\lim _{k} \inf \left(b, y_{k}\right):\left\{y_{k}, z_{k}\right\} \in A F(2)\right\}>-\infty\right] .
$$

Otherwise it is AUBD (asymptotically UBD).

1. The duality states. Of the 49 mutually exclusive and collectively exhaustive states for problems (I.C) and (II.C*) only 10 are possible, and are those denoted in the table below by positive integers. A zero in the table means that the corresponding state is impossible. This classification constitutes our theorem, whose proof is given in two parts. Part 1 proves the possible states by examples so numbered that the possibility of each state is demonstrated by the example bearing the same number. Part 2 excludes the impossible states by a series of lemmas.

2 In order to refine and extend the original cases developed by Duffin [3], we take the liberty of deviating from his terminology. 
THEOREM.

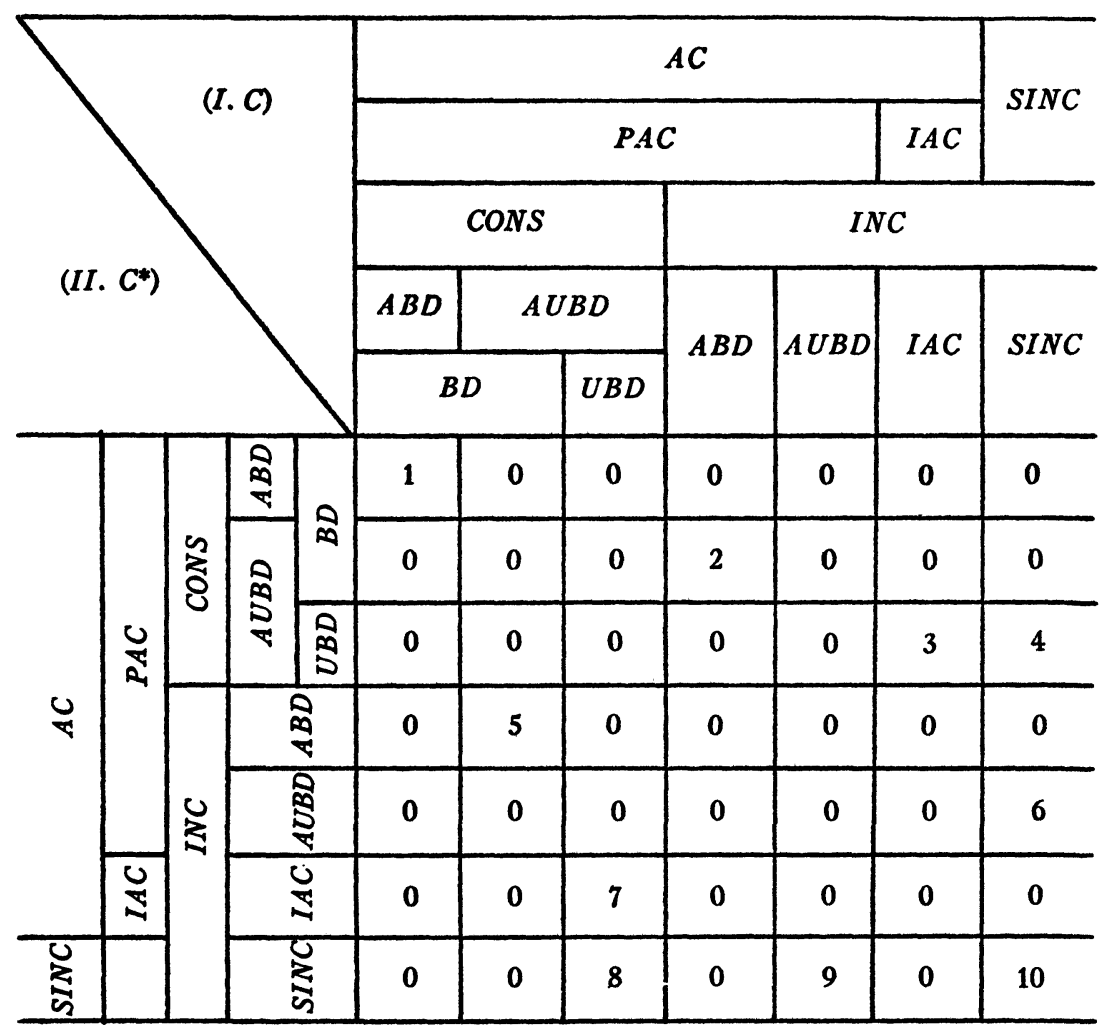

Proof. Part 1. The possible states:

Let $C$ be the cone in $R^{3}$ of all vectors forming an angle $\leqq 45^{\circ}$ with the vector $(1,0,1)$. Then $C=C^{*}$. In all 10 examples below we use this $C$ and one of the following matrices

for $A$.

$$
A_{1}=\left(\begin{array}{lll}
0 & 1 & 0 \\
0 & 0 & 1
\end{array}\right), \quad A_{2}=\left(\begin{array}{lll}
0 & 0 & 1
\end{array}\right)
$$

Example 1. $A=A_{1}, b=(0,1), c=(0,0,1)$,

Example 2. $A=A_{1}, b=(1,0), c=(0,0,0)$,

Example 3. $A=A_{1}, b=(1,0), c=(-1,0,0)$,

Example 4. $A=A_{2}, b=(-1), c=(-1,0,0)$,

Example 5. $A=A_{2}, b=(0), c=(0,-1,0)$,

Example 6. $A=A_{2}, b=(-1), c=(0,1,0)$,

Example 7. $A=A_{2}, b=(1), c=(0,1,0)$,

Example 8. $A=A_{2}, b=(1), c=(1,0,0)$, 
Example 9. $A=A_{1}, b=(1,0), c=(1,0,0)$,

Example 10. $A=A_{2}, b=(-1), c=(1,0,0)$.

Part 2. The impossible states:

LemMA 1. (I.C) $A C$ and (II.C*) $C O N S \Rightarrow$

(3) $\sup \left\{\lim _{z} \sup \left(c, x_{k}\right):\left\{x_{k}\right\} \in A F(1)\right\} \leqq \inf \{(b, y):(y, z) \in F(2)\}$.

Moreover if (II.C*) is also BD then $\exists$ a sequence $\left\{x_{k}\right\} \in A F(1) \ni$

$$
\lim _{c} \sup \left(c, x_{k}\right)=\inf \{(b, y):(y, z) \in F(2)\} \text {. }
$$

Proof. To prove (3) let $\left\{x_{k}\right\} \in A F(1)$ and $A^{t} y-c=z \in C^{*}$. Then

$$
\begin{aligned}
(b, y) & =\lim _{k}\left(A x_{k}, y\right)=\lim _{k}\left(x_{k}, A^{t} y\right)=\lim _{k}\left(x_{k}, c+z\right) \\
& =\lim _{k} \sup \left(x_{k}, c+z\right) \\
& \geqq \lim _{k} \sup \left(x_{k}, c\right) \quad \text { since } x_{k} \in C, z \in C^{*},
\end{aligned}
$$

which proves (3).

To prove the existence of a maximizing sequence $\left\{x_{k}\right\} \in A F(1)$ let

$$
\beta=\inf \{(b, y):(y, z) \in F(2)\} .
$$

Since (II.C*) is BD it follows that

$$
\left(\begin{array}{rrr}
A^{t} & -I & -c \\
0 & I & 0 \\
0 & 0 & 1
\end{array}\right)\left(\begin{array}{c}
y \\
z \\
z_{n+1}
\end{array}\right) \in\{0\} \times C^{*} \times R_{+}
$$

$$
\Rightarrow\left(\left(\begin{array}{c}
b \\
0 \\
-\beta
\end{array}\right),\left(\begin{array}{c}
y \\
z \\
z_{n+1}
\end{array}\right)\right) \geqq 0 \text {. }
$$

For if $z_{n+1}>0$ then by setting $z_{n+1}=1$ (6) follows from the definition (5) of $\beta$. If on the other hand $z_{n+1}=0$ and (6) is false, i.e. $\exists\left(y^{0}, z^{0}\right) \ni$

$$
\left(\begin{array}{rrr}
A^{t} & -I & -q \\
0 & I & 0 \\
0 & 0 & 1
\end{array}\right)\left(\begin{array}{c}
y^{0} \\
z^{0} \\
0
\end{array}\right) \in\{0\} \times C^{*} \times R_{+}, \quad\left(\left(\begin{array}{c}
b \\
0 \\
-\beta
\end{array}\right),\left(\begin{array}{l}
y^{0} \\
z^{0} \\
0
\end{array}\right)\right)<0,
$$

then for any $(y, z) \in F(2)$ we take $\left(y_{k}, z_{k}\right)=(y, z)+k\left(y^{0}, z^{0}\right), k=1$, $2, \cdots$ and verify that

$$
\left(y_{k}, z_{k}\right) \in F(2) \quad \forall k .
$$


But $\left(b, y_{k}\right) \rightarrow-\infty$, which contradicts (II.C*) BD. Finally (6) is equivalent to

$$
\left(\begin{array}{ccc}
A & 0 & 0 \\
-I & I & 0 \\
-c^{t} & 0 & 1
\end{array}\right)\left(\begin{array}{l}
x \\
u \\
v
\end{array}\right)=\left(\begin{array}{c}
b \\
0 \\
-\beta
\end{array}\right), \quad\left(\begin{array}{l}
x \\
u \\
v
\end{array}\right) \in R^{n} \times C \times R_{+}
$$

being AC, which proves the existence of $\left\{x_{k}\right\} \in A F(2)$ satisfying (4).

Lemma 2. (I.C) CONS and (II.C*)

$$
A C \Rightarrow \sup \{(c, x): x \in F(1)\} \leqq \inf \left\{\liminf _{k}\left(b, y_{k}\right):\left\{y_{k}, z_{k}\right\} \in A F(2)\right\} \text {. }
$$

Moreover if (I.C) is also BD then $\exists$ a sequence

$$
\left\{y_{k}, z_{k}\right\} \in A F(2) \ni \sup \{(c, x): x \in F(1)\}=\liminf _{k}\left(b, y_{k}\right) .
$$

Lemma 3. (I.C) $P A C$ and $A B D \Rightarrow($ II.C*) CONS and $B D$.

Lemma 4. (II.C*) $P A C$ and $A B D \Rightarrow$ (I.C) CONS and $B D$.

LEMma 5. One of the problems (I.C) and (II.C*) is CONS and ABD iff so is the other, in which case

$$
\begin{aligned}
& \sup \left\{\limsup _{k}\left(c, x_{k}\right):\left\{x_{k}\right\} \in A F(1)\right\}=\inf \{(b, y):(y, z) \in F(2)\} \\
& \geqq \inf \left\{\lim _{k} \inf \left(b, y_{k}\right):\left\{y_{k}, z_{k}\right\} \in A F(2)\right\}=\sup \{(c, x): x \in F(1)\} .
\end{aligned}
$$

LEMMA 6. (I.C) $I A C \Rightarrow$ (II.C*) CONS and UBD.

Proof. We prove first that (I.C) IAC $\Rightarrow($ II.C*) CONS. Assume to the contrary that (II.C*) is INC. Then for any $r>0$

$$
\left(A^{t},-c\right)\left(\begin{array}{c}
y \\
y_{m+1}
\end{array}\right) \in C^{*} \Rightarrow\left(\left(\begin{array}{c}
y \\
y_{m+1}
\end{array}\right),\left(\begin{array}{c}
0 \\
-r
\end{array}\right)\right) \geqq 0 .
$$

By the solvability theorem this is equivalent to

$$
\left(\begin{array}{c}
A \\
-c^{t}
\end{array}\right) u=\left(\begin{array}{c}
0 \\
-r
\end{array}\right), \quad u \in C
$$

being AC.

Let now $\left\{x_{k}\right\} \in A F(1)$ and

$$
r_{k}=\left|\left(c, x_{k}\right)\right| \quad k=1,2, \cdots .
$$

For each $k$ let $\left\{u_{k j}\right\} \in A F\left(8 . r_{k}\right)$ of which we choose an element 
satisfying

$$
\left|\frac{r_{k} A u_{k j(k)}}{\left(c, u_{k j(k)}\right)}\right|<\frac{1}{2^{k}}, \quad k=1,2, \cdots
$$

The sequence

$$
\left\{v_{k}\right\}=x_{k}+r_{k} \frac{u_{k j(k)}}{\left(c, u_{k j(k)}\right)}, \quad k=1,2, \cdots,
$$

satisfies

$$
\left\{v_{k}\right\} \in A F(1)
$$

but

$$
\left(c, v_{k}\right)=\left(c, x_{k}\right)+\left|\left(c, x_{k}\right)\right| \geqq 0
$$

contradicting (I.C) IAC. Finally (II.C*) is UBD. For if it is BD then by Lemma 1 (I.C) cannot be IAC.

LEMMA 7. (II.C*) $I A C \Rightarrow($ I.C) $C O N S$ and $U B D$.

The zero-numbered states in the above table are excluded by applying Lemmas 1-7. This completes the proof of the theorem.

2. Remarks. (i) If the cone $C$ (hence $C^{*}$ ) is polyhedral then $F(1)=A F(1)$ and $F(2)=A F(2)$ so that both problems (I.C) and (II.C*) are either CONS or SINC. Only the 4 states, nos. 1, 4, 8 and 10 , are then possible. For $C$ polyhedral our results thus give (an alternate proof of) the duality theorem of linear programming, e.g. [4], [2].

(ii) The following simple example shows the possibility of arbitrary nonnegative values for the difference between the sides of inequality (7) in Lemma 5.

Let $C=C^{*}$ be the cone given in Part 1 of the proof and let

$$
\bar{C}=C \times R_{+}=C^{*} .
$$

For any $\alpha \leqq 0$, let the problem (I. $\tilde{C})$ be given as

$$
\begin{array}{lrr}
\begin{array}{l}
\operatorname{maximize}-x_{2} \\
\text { s.t. }
\end{array} & =0 \\
& x_{2} & -x_{4}=\alpha \\
\left(x_{1}, x_{2}, x_{3}, x_{4}\right) \in \tilde{C} . &
\end{array}
$$


The problem (II. $\left.\tilde{C}^{*}\right)$ is

$$
\begin{aligned}
& \operatorname{minimize} \alpha y_{2} \\
& \text { s.t. } \quad\left(0,1+y_{2}, y_{1},-y_{2}\right) \in \tilde{C} .
\end{aligned}
$$

Both problems are CONS and ABD. Furthermore

$\sup \left\{\lim \sup \left(c, x_{k}\right):\left\{x_{k}\right\} \in A F(1)\right\}=\inf \{(b, y):(y, z) \in F(2)\}=-\alpha$ and

$\sup \{(c, x): x \in F(1)\}=\inf \left\{\liminf _{k}\left\{\left(b, y_{k}\right):\left\{y_{k}, z_{k}\right\} \in A F(2)\right\}\right\}=0$.

The difference in (7) is thus $-\alpha$.

(iii) Lemmas 1,2 and 5 reprove Duffin's duality theorem (1 of $[3])$.

(iv) The above results show that if both problems (I.C) and (II.C*) are INC, then at least one of them is SINC. It is thus impossible for both problems to be AC but INC.

\section{REFERENCES}

1. A. Ben-Israel and A. Charnes, On the intersection of cones and subspaces, Bull. Amer. Math. Soc. 74 (1968), 541-544.

2. A. Charnes, W. W. Cooper and K. Kortanek, A duality theory for convex programs with convex constraints, Bull. Amer. Math. Soc. 68 (1962), 605-608.

3. R. J. Duffin, "Infinite programs" in Linear inequalities and related systems, edited by H. W. Kuhn and A. W. Tucker, Ann. of Math. Studies No. 38, Princeton Univ. Press, Princeton, N. J., 1956, pp. 157-170.

4. D. Gale, H. W. Kuhn and A. W. Tucker, "Linear programming and the theory of games" in Cowles commission for research in economics, edited by T. C. Koopmans, Monograph No. 13, Wiley, New York, 1951, pp. 317-329.

5. K. S. Kretschmer, Programmes in paired spaces, Canad. J. Math. 13 (1961), 221-238.

NORTHWESTERN UNIVERSITY, EvaNSTON, IllinoIs 60201 AND

Corneli. University, Ithaca, New York 14850 